\title{
Arginine Vasopressin Injected into the Dorsal Motor Nucleus of the Vagus Inhibits Gastric Motility in Rats
}

\author{
Jianping Zhu, Lanlan Chang, Jinlu Xie, and Hongbin Ai \\ College of Life Sciences, Shandong Normal University, Jinan 250014, China \\ Correspondence should be addressed to Hongbin Ai; aihb518@126.com
}

Received 1 August 2015; Revised 25 October 2015; Accepted 27 October 2015

Academic Editor: Guillaume Gourcerol

Copyright (c) 2016 Jianping Zhu et al. This is an open access article distributed under the Creative Commons Attribution License, which permits unrestricted use, distribution, and reproduction in any medium, provided the original work is properly cited.

\begin{abstract}
Background. Until now, the effect of arginine vasopressin (AVP) in the DMV on gastric motility and the possible modulating pathway between the DMV and the gastrointestinal system remain poorly understood. Objectives. We aimed to explore the role of AVP in the DMV in regulating gastric motility and the possible central and peripheral pathways. Material and Methods. Firstly, we microinjected different doses of AVP into the DMV and investigated its effects on gastric motility in rats. Then, the possible central and peripheral pathways that regulate gastric motility were also discussed by microinjecting SR49059 (a specific AVP receptor antagonist) into the DMV and intravenous injection of hexamethonium (a specific neuronal nicotinic cholinergic receptor antagonist) before AVP microinjection. Results. Following microinjection of AVP (180 pmol and 18 pmol) into the DMV, the gastric motility (including total amplitude, total duration, and motility index of gastric contraction) was significantly inhibited $(P<0.05)$. Moreover, the inhibitory effect of AVP (180 pmol) on gastric motility could be blocked completely by both SR49059 (320 pmol) and hexamethonium $(8 \mu \mathrm{mol})$. Conclusions. It is concluded that AVP inhibits the gastric motility by acting on the specific AVP receptor in the DMV, with the potential involvement of the parasympathetic preganglionic cholinergic fibers.
\end{abstract}

\section{Introduction}

Arginine vasopressin (AVP) is synthesized by the vasopressinergic neurons of paraventricular hypothalamic nucleus (PVN) and supraoptic nucleus (SON) and plays important roles in water conservation, and so on. Recent studies have demonstrated that AVP is synthesized throughout the brain and can function as a neurotransmitter/neuromodulator and modulate other complicated and varying autonomic function, not just antidiuresis and vascular tone [1-5]. The dorsal motor nucleus of the vagus (DMV) primarily serves parasympathetic functions and consists largely of vagal preganglionic neurons that project to the stomach and control gastric motility [6]. Numerous anatomical and electrophysiological studies have provided evidence for direct and monosynaptic projections from the PVN to the DMV [7-9], which indicates that afferent information from the PVN to the DMV might participate in the process of modulating activities of neurons in the DMV.

In our previous studies, we had noted that waterimmersion restraint-related stress in rats activated both vasopressinergic and oxytocinergic neurons in the PVN and caused serious gastric dysfunction. Meanwhile there were AVP and oxytocin (OT) receptors largely expressed in the soma and/or dendrite membranes of the activated neurons in the DMV $[10,11]$, suggesting the possible involvement of vasopressinergic/oxytocinergic projections from the PVN to the DMV in modulating the gastric functions.

The long-descending oxytocinergic projection from the PVN to the DMV has been reported to serve as a link in the main neural control of the gastric function, indicating the important role of OT in autonomic function. The gastricrelated neurons in the DMV have been shown to belong to OT-sensitive neurons [12], which is involved in regulating gastric motility $[1,13]$. AVP and OT are regarded as highly related nonapeptides hormones in structure and could combine with their receptors reciprocally [14], indicating that AVP might also be a central regulator of gastric function. However, none has been done so far to investigate the relationship between AVP in the DMV and gastric function, and whether changes in DMV neuron activity initiated by AVP produce a modulation of gastric motility is still not 
TABLE 1: The design of the experiment.

\begin{tabular}{|c|c|c|c|c|c|c|}
\hline Group & Numbers & Chemicals & Dosage or concentration & Volume & Sites of injection & Detecting index \\
\hline Group 1 & 6 & AVP & $180 \mathrm{pmol}$ & $50 \mathrm{~nL}$ & DMV & $\mathrm{GM}, \mathrm{BP}, \mathrm{HR}$, and RF \\
\hline Group 2 & 6 & AVP & $18 \mathrm{pmol}$ & $50 \mathrm{~nL}$ & DMV & $\mathrm{GM}, \mathrm{BP}, \mathrm{HR}$, and RF \\
\hline Group 3 & 6 & NS & $0.9 \%$ & $50 \mathrm{~nL}$ & DMV & $\mathrm{GM}, \mathrm{BP}, \mathrm{HR}$, and RF \\
\hline Group 4 & 6 & $\begin{array}{c}\text { SR49059 } \\
\text { AVP }\end{array}$ & $\begin{array}{l}320 \mathrm{pmol} \\
180 \mathrm{pmol}\end{array}$ & $50 \mathrm{~nL}$ & DMV & GM \\
\hline Group 5 & 6 & $\begin{array}{c}\text { Hexamethonium } \\
\text { AVP }\end{array}$ & $\begin{array}{c}8 \mu \mathrm{mol} \\
180 \mathrm{pmol}\end{array}$ & $\begin{array}{l}1 \mathrm{~mL} \\
50 \mathrm{~nL}\end{array}$ & $\begin{array}{c}\text { Femoral Vein } \\
\text { DMV }\end{array}$ & GM \\
\hline
\end{tabular}

GM, gastric motility; BP, blood pressure; HR, heart rate; RF, respiratory frequency; NS, normal saline.

well defined yet. Thus, the present study aimed to investigate whether AVP in the DMV plays a role in regulating the gastric motility. Additionally, the central actions of AVP are mainly mediated by V1 receptors [15], and most of the vagal preganglionic neurons controlling gastric function in the DMV are cholinergic neurons in nature [16]. Therefore, the possible central and peripheral mechanism of AVP on gastric motility were also explored by using AVP receptor blocker (SR49059) and preganglionic antagonist (Hexamethonium).

\section{Materials and Methods}

\subsection{Materials}

2.1.1. Chemicals. Arginine vasopressin (AVP), SR49059, neutral red, and Hexamethonium were purchased from Sigma, USA, and chloral hydrate and $\mathrm{NaCl}$ were purchased from Tiancheng, Chemical Ltd., China. All chemicals were in technical grade of more than 95\% purity. All drugs were dissolved in normal saline (NS) and prepared immediately before use.

2.1.2. Animals. Male adult Wistar rats (a total of 30, 280$320 \mathrm{~g}$ body weight, 3 months old) were graciously provided by the Experimental Animal Center of Shandong University, China. Before experiments, all test animals were kept in a temperature-controlled environment on 12-hour light and 12hour darkness at $22 \pm 1^{\circ} \mathrm{C}$. Prior to experiments, rats were fasted for 24 hours, and water was provided ad libitum. All procedures were conducted in accordance with the National Institutes of Health Guidelines for the Care and Use of Laboratory Animals and were approved by the Institutional Animal Care and Use Committee at Shandong Normal University.

2.2. Methods. Rats were selected randomly and divided into five groups, and the experimental design is shown in Table 1.

2.2.1. Recording of Gastric Motility. Animals were anesthetized with an intraperitoneal (i.p.) injection of sodium chloral hydrate ( $400 \mathrm{mg} / \mathrm{kg}$ body mass) and were placed on a small animal operating table in the supine position. A midline laparotomy was performed, and a latex balloon filled with water ( $5 \mathrm{~mm}$ in diameter) was inserted into the pyloric region through a small incision in the forestomach wall. The balloon was connected to a pressure transducer via a thin polyethylene tube. Blood pressure (BP) was monitored via another pressure transducer connected via a cannula inserted into the femoral artery. After the above procedures were completed, the rats were placed on a stereotaxic apparatus; body temperature was kept constant at $37 \pm 1^{\circ} \mathrm{C}$ with a radiant heat lamp. The respiratory frequency $(\mathrm{RF})$ was monitored by a tonotransducer attached to the rat's chest with a clamp. Heart activity was monitored via electrocardiogram (ECG), which was recorded synchronously via the standard leads connected to all four limbs. All the physiological signals were fed into the Biological Function Experiment System (BL-420, Taimeng Technical Co., Chengdu, China). Gastric motility (GM) was recorded for at least $30 \mathrm{~min}$ before microinjection and lasted for $30 \mathrm{~min}$ after microinjection. The recording of BP, RF, and ECG was made simultaneously with gastric motility.

2.2.2. Stereotaxis and Microinjection. The rats were placed on a stereotaxic apparatus (Stoelting 51600, USA), and the dorsal surface of the medulla was exposed through an occipital craniotomy. A glass micropipette $(30-50 \mu \mathrm{m}$, external tip diameter) was connected with a microsyringe (volume $1 \mu \mathrm{L}$, Anting Scientific Instrument Company, Shanghai, China) via polyethylene tube filled with NS. After removal of the dura, the micropipette tip was stereotaxically introduced into the DMV according to the atlas of Paxinos and Watson (5th edition, coordinates: $0.5-0.9 \mathrm{~mm}$ rostral to the obex, a depth of $0.4-0.5 \mathrm{~mm}$ below the surface of the obex, $0.5 \mathrm{~mm}$ lateral to the midline). The microinjection of AVP into the DMV was performed in a volume of $50 \mathrm{~nL}$ for $10-15 \mathrm{sec}$. In order to investigate the possible central effect of AVP in the DMV on gastric function, a specific V1 receptor antagonist SR49059 was also administered to rats before each microinjection of AVP. Hexamethonium (autonomic ganglionic cholinergic receptor blockade) was also delivered via intravenous injection before the microinjection of AVP, to explore the possible peripheral mechanisms of AVP on gastric function.

2.2.3. After Treatment. At the end of the experiment, $2 \%$ potamine sky blue $(50 \mathrm{~nL})$ was injected into the same microinjection site. The rats were then deeply anesthetized by intraperitoneal injections of sodium pentobarbital $(100 \mathrm{mg} / \mathrm{kg}$ body weight) and were perfused transcardially with NS followed by $4 \%$ paraformaldehyde. The brains were removed and fixed in $4 \%$ paraformaldehyde with $20 \%$ sucrose for 2 days. The bulbar region was frozen, serially sectioned 


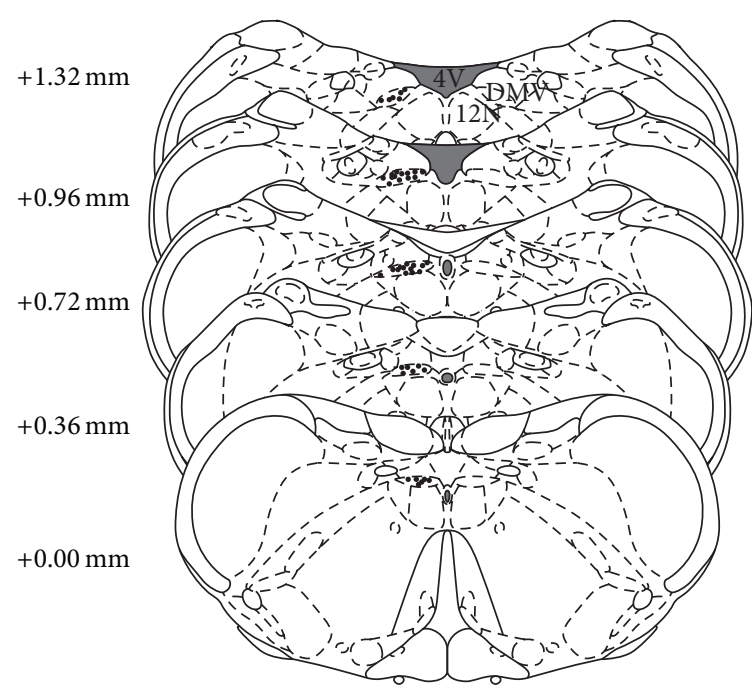

(a)

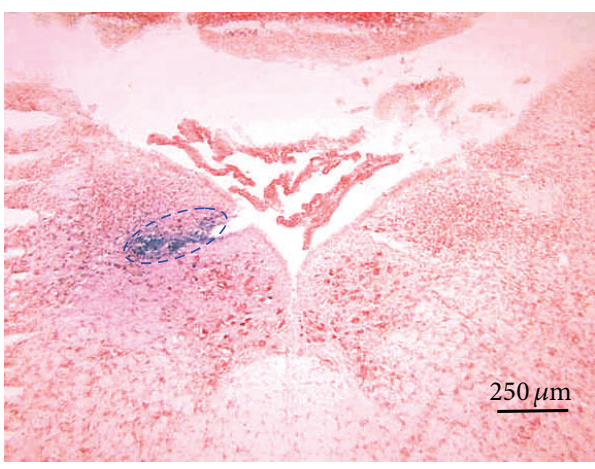

(b)

FIgURE 1: Histological sites of micropipette tips in the DMV of the rat. Reconstruction detailing microinjection sites of chemical into the DMV in the brain atlas, with the dots indicating the micropipette tips. Numbers indicate distance from the obex in the anteroposterior plane. $4 \mathrm{~V}$ : the 4 th ventricle, $12 \mathrm{~N}$ : the hypoglossal nucleus (a). Representative brain stem section showing the position of DMV, with ellipse and blue macula indicating DMV (b).

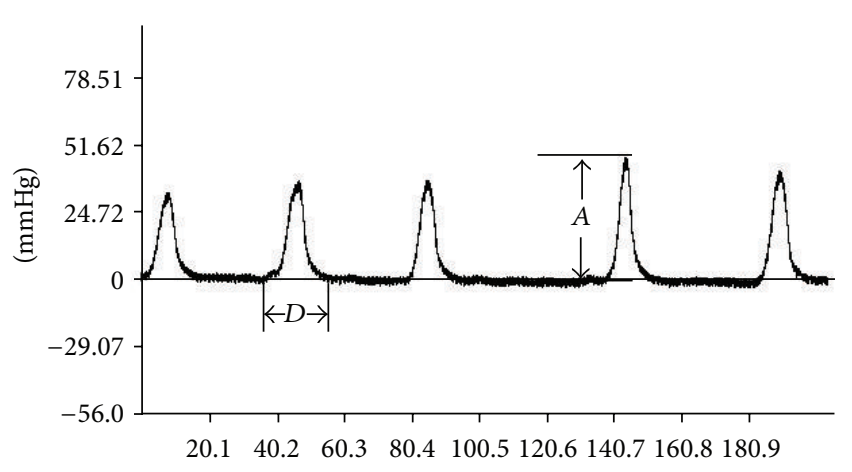

(s)

FIGURE 2: Representative gastric contraction wave in rat, showing the duration $(D)$ and amplitude $(A)$.

$(40 \mu \mathrm{m})$, and stained with neutral red to facilitate the identification of the micropipette tip in the DMV (Figure 1). All the tip sites of the microinjection were identified, and the data from the rats with the tip sites located outside the DMV were excluded in the analysis. Images of brain sections were taken under identical conditions with a microscope (Olympus Co., Japan) coupled with a digital camera.

2.3. Data Analysis. Total duration (the sum of wave durations per $5 \mathrm{~min}$ ), total amplitude (the sum of wave amplitudes per $5 \mathrm{~min}$ ), and motility index (MI) of gastric contraction waves within $5 \mathrm{~min}$ before microinjection and three consecutive $5 \mathrm{~min}$ values after microinjection were measured. In this study, the duration of each contraction wave was defined as the timespan between the onset of ascending phase and the offset of descending phase from the baseline, and the amplitude was defined as the altitude of each contraction wave (Figure 2). The motility index was defined as the product of amplitude and duration of each contraction wave. Inhibitory rates (IR) were applied to estimate the changing degree of gastric motility before and after microinjection; namely, IR $(\%)=100 \% \times($ the value before microinjection the value after microinjection)/the value before microinjection. Changes in BP (mmHg, mean arterial pressure), RF (times/min), and HR (beats/min) were monitored before and after microinjection for $60 \mathrm{sec}$.

Variables were analyzed using SPSS 13.0 software (SPSS Inc., Chicago, IL, USA) and presented as mean \pm SEM. Data were analyzed using one-way analysis of variance followed by Student-Newman-Keuls multiple-comparisons or Student's $t$-test. Statistical significance was set at $P<0.05$.

\section{Results}

3.1. Effects of Different Doses of AVP on Gastric Motility. As shown in Figure 3, the microinjection of AVP (180 pmol or $18 \mathrm{pmol}$ ) into the DMV caused a significant inhibition of gastric motility within 5 min $(P<0.05)$ in groups 1 and 2 (Figures $3(\mathrm{a})-3(\mathrm{c})$ ). The inhibitory rates of AVP in group 1 microinjected into the DMV (the first $5 \mathrm{~min}$ after microinjection) for total duration, total amplitude, and motility index were $56.50 \%, 53.15 \%$, and $56.08 \%$, respectively. These percentages were higher than those of AVP in group 2, which suggested that the inhibitory effect of AVP on gastric motility showed a dose-dependent trend in the first $5 \mathrm{~min}$ after microinjection (Figures 3(d)-3(f)). In the following 5 minutes in group 1 and group 2, the inhibition of the gastric motility decreased gradually. Ten minutes later after microinjection of AVP, gastric contraction waves returned to normal. No significant inhibition of gastric motility was 


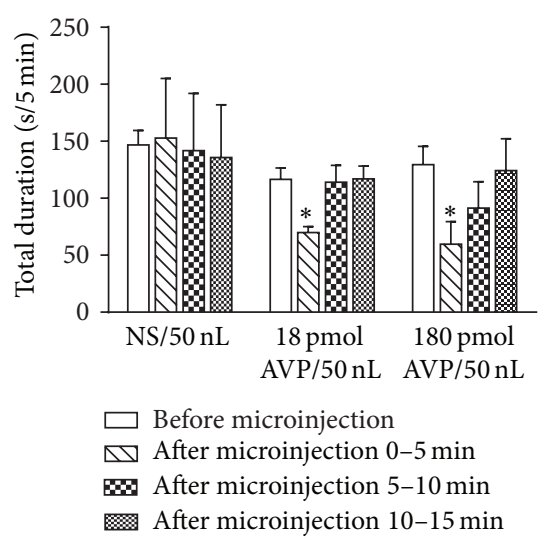

(a)

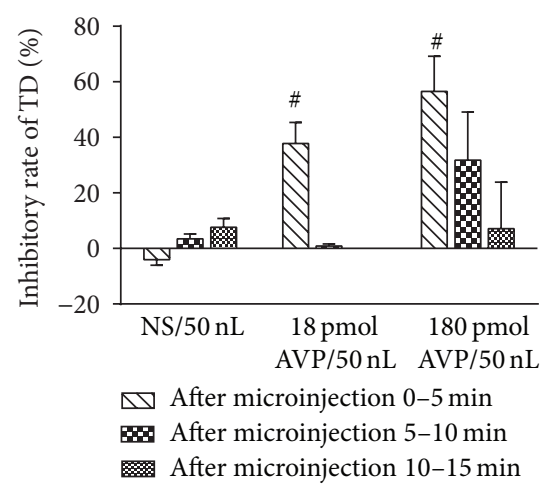

(d)

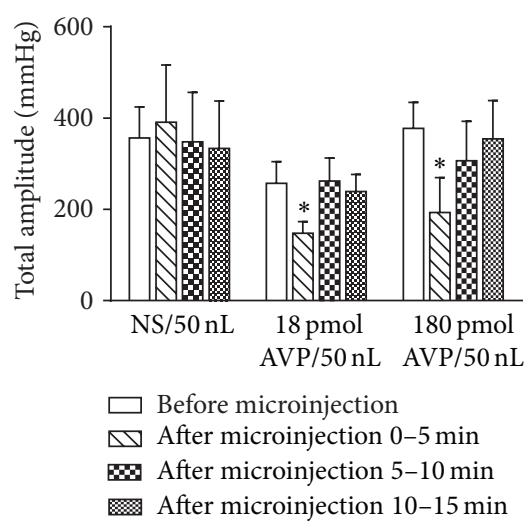

(b)

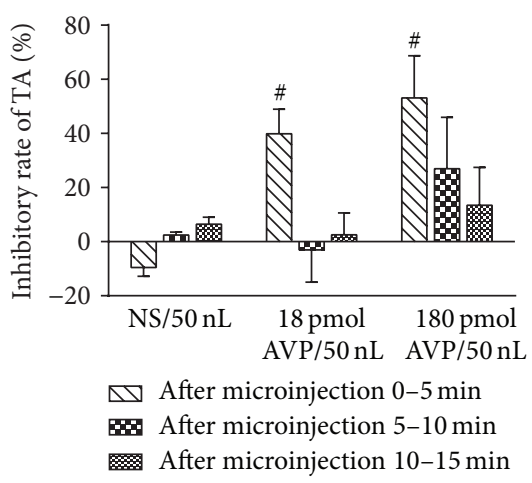

(e)

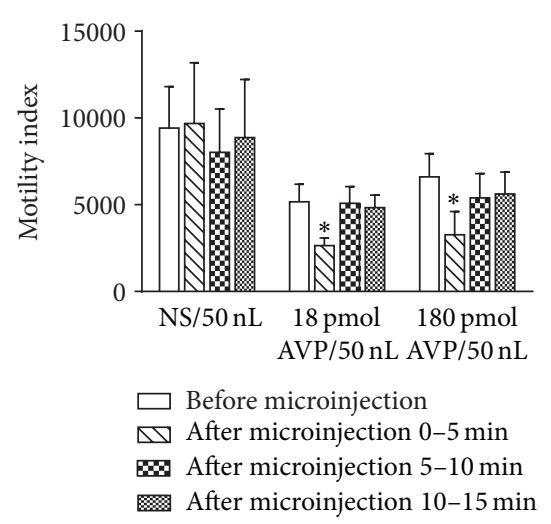

(c)

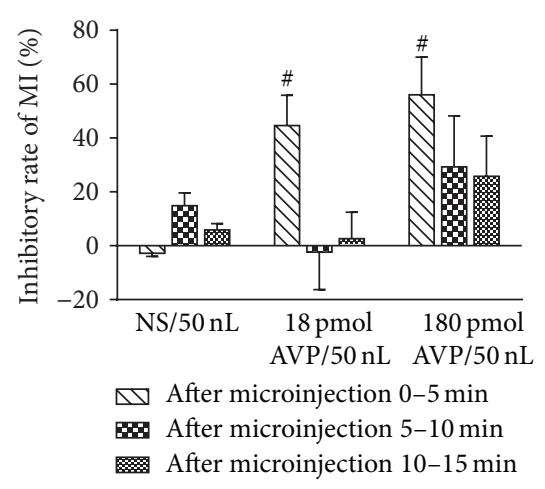

(f)

FIGURE 3: Representative data for gastric motility before and after microinjection of AVP (18 and 180 pmol) or normal saline (NS) into the DMV. Mean data of total duration (a), total amplitude (b), and motility index (c) before microinjection and after microinjection 0-5 min, 5-10 min, and 10-15 min. Average inhibitory rate of total duration (d), total amplitude (e), and motility index (f) in the first $5 \mathrm{~min}$, second $5 \mathrm{~min}$, and third 5 min. ${ }^{*} P<0.05$, versus before microinjection; ${ }^{\#} P<0.05$, versus NS group in the same period.

observed in group 3, in which AVP was substituted for NS $(P>0.05)$.

As shown in Figure 4, the microinjection of $180 \mathrm{pmol}$ AVP in group 1 significantly decreased the respiratory frequency and the heart rate (after microinjection, $P<0.01$ or $P<0.05$ ). The inhibitory rates of $180 \mathrm{pmol}$ AVP on the respiratory frequency and the heart rate were $23.99 \%$ and $28.26 \%$, respectively. However, in group 2 or group 3, the administration of NS or 18 pmol AVP on the respiratory frequency and the heart rate resulted in no significant difference $(P>0.05)$. Blood pressure in three groups was similar.

3.2. Effects of AVP on Gastric Motility with Pretreatment of SR49059 or Hexamethonium. In group 4, 320 pmol SR49059 was microinjected into the DMV of normal rats, and gastric motility was stimulated within $5 \mathrm{~min}(P<0.05 ; P<$ 0.01) (Figures 5(a)-5(c)). The inhibitory rates of SR49059 for total duration, total amplitude, and motility index were $-38.28 \%,-48.47 \%$, and $-53.14 \%$, respectively (Figure 5(d)). After $15 \mathrm{~min}, 180 \mathrm{pmol}$ AVP was delivered into the same site to investigate the effect of AVP on gastric motility in the presence of SR49059. The results showed that AVP had no effect on gastric motility in the presence of SR49059 (Figures 5(a) $-5(\mathrm{c})$ and 5(e)).
In group 5, Hexamethonium was delivered via intravenous injection through the femoral vein in normal rats, and gastric motility was completely inhibited $(P<0.01)$ (Figures 5(a)-5(c)). The inhibitory rates of total duration, total amplitude, and motility index were $98.43 \%, 99.26 \%$, and $99.96 \%$, respectively (Figure 5(d)). Half an hour later, gastric contraction waves were restored, but at a relatively stable, low amplitude state. $180 \mathrm{pmol}$ AVP was microinjected into the DMV and no change was observed (Figure 5(e)).

\section{Discussion}

4.1. Effects of AVP on Gastric Motility. It is well documented that the PVN is a central nucleus for modulating gastric function. Meanwhile a growing body of evidence on the morphology of PVN-DMV projection has shown that the vasopressinergic fibers from PVN project directly to the DMV $[7,9]$, suggesting a close relationship between AVP in the DMV and gastric function. In this study, in group 1 or group 2, the microinjection of AVP (180 pmol or $18 \mathrm{pmol})$ into the DMV produced an inhibition on gastric motility in a dose-dependent manner within $5 \mathrm{~min}$, suggesting that extrinsic AVP participates in modulating the gastric motility. In group 4, SR49059, a specific V1 receptor antagonist, 


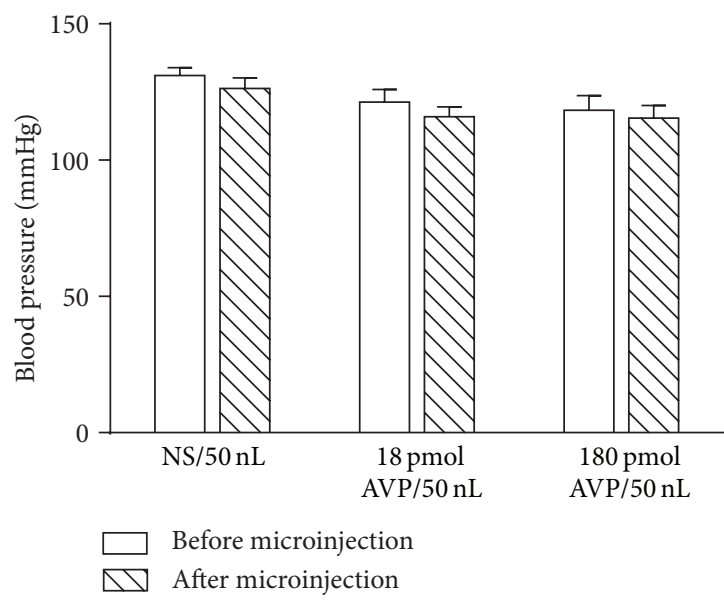

(a)

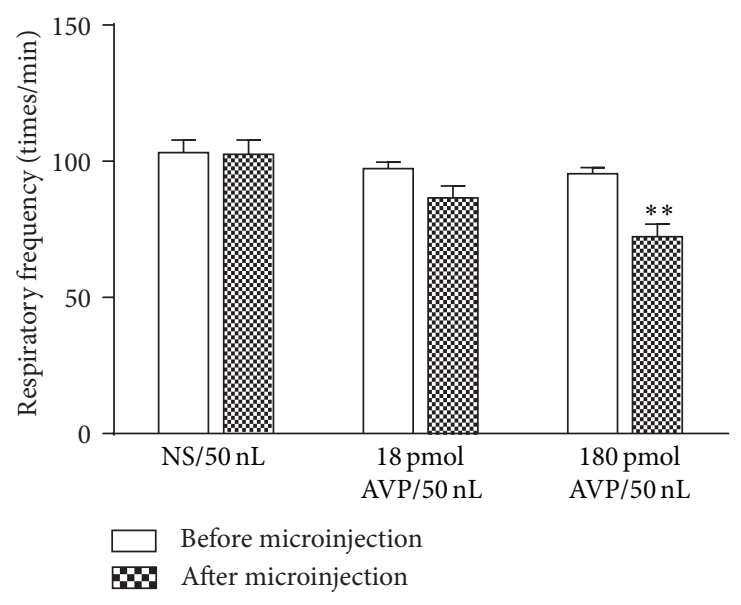

(c)

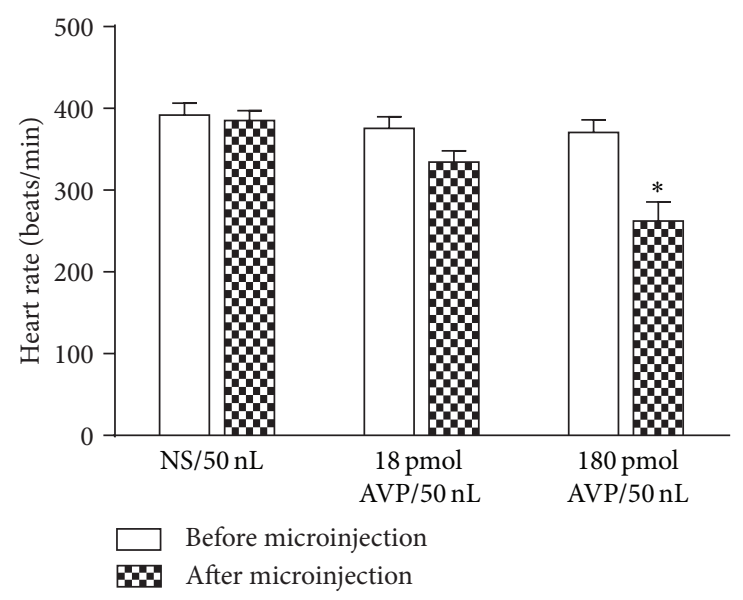

(b)

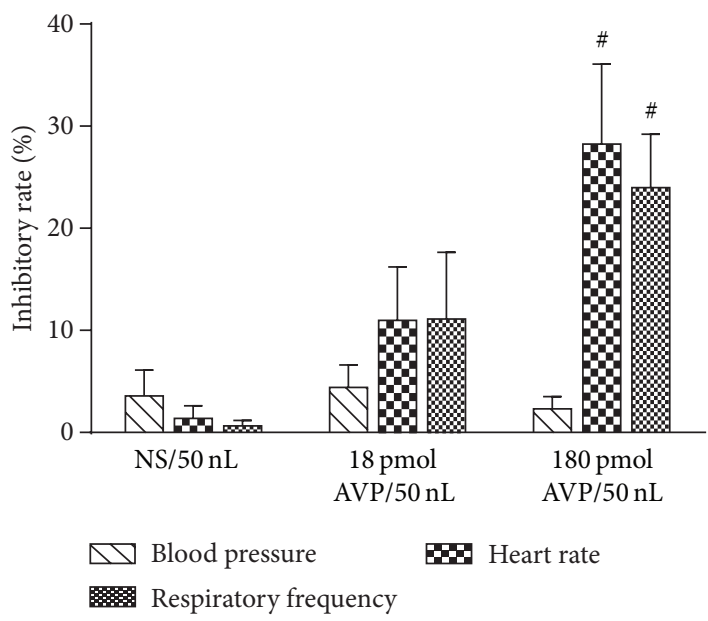

(d)

FIgURE 4: Effects of AVP (18 and 180 pmol) or normal saline (NS) microinjected into the DMV on blood pressure, heart rate, and respiratory frequency. Representative effects of AVP or NS on blood pressure (a) and heart rate (b) and respiratory frequency (c) before and after microinjection. The inhibitory rate of AVP or NS on blood pressure and heart rate and respiratory frequency $(\mathrm{d}) .{ }^{*} P<0.05$ and ${ }^{* *} P<0.01$, versus before microinjection; ${ }^{\#} P<0.05$, versus NS group in the same period.

injected into the DMV of normal rats significantly increased gastric motility within $5 \mathrm{~min}$, suggesting the involvement of intrinsic AVP from PVN in modulating the gastric motility. In group 2, 18 pmol AVP inhibited the gastric motility but had no effect on the respiratory frequency, the heart rate, and blood pressure, suggesting that the effect of AVP on gastric motility was specific and not due to a global depression linked to systemic action. All findings revealed that AVP participated in the process of modulating gastric motility by activating neurons of DMV, not by systemic action.

Growing evidence indicates the involvement of OT exciting gastric-related neurons of the DMV in modulating the gastric function [17-21]. Despite much research in OT from a clinical and experimental perspective, little is known about whether AVP in the DMV are involved in the process of modulating gastric motility in vivo in rats. To our knowledge, there has been no such study so far. AVP and OT are the highly related nonapeptides in structure released by PVN and SON, showing only a little difference in two of all the amino acids. AVP and OT activate the neurons via G-protein coupled receptor and thus could combine with their receptors reciprocally [14]. Our present data strongly demonstrate that the vasopressinergic projection from the PVN to the DMV probably plays a similar role in the gastric functions and produces an excitatory effect on the neurons of DMV as the oxytocinergic system does.

The DMV contains cell bodies of the vagal preganglionic neurons that could control gastric motility, and the vast majority $(>95 \%)$ of neurons innervating the stomach are cholinergic [22]. Microinjection of L-Glutamate into the rostral or caudal DMV could produce gastric contraction or relaxation, respectively, suggesting a characteristic spatial organization of vagal preganglionic neurons in the DMV $[16,23,24]$. Cruz et al. reported that the vagal efferent pathway which could excite gastric motility was originated from the area of the DMV rostral to calamus scriptorius (CS) and which could inhibit gastric motility was originated from the area of the DMV caudal to CS [25]. However, in the present 


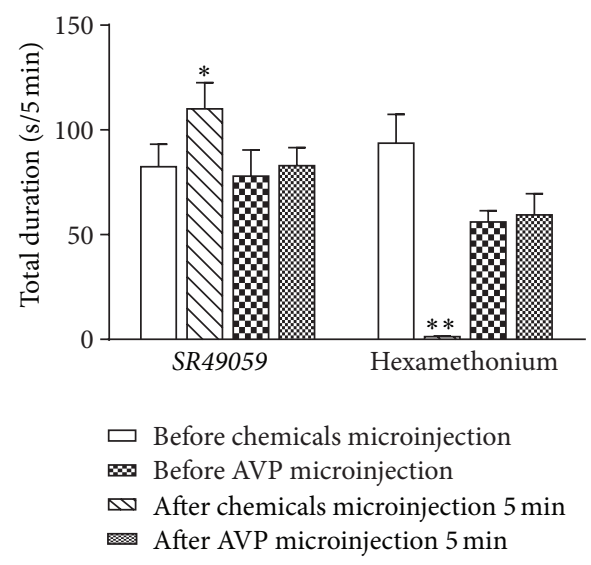

(a)

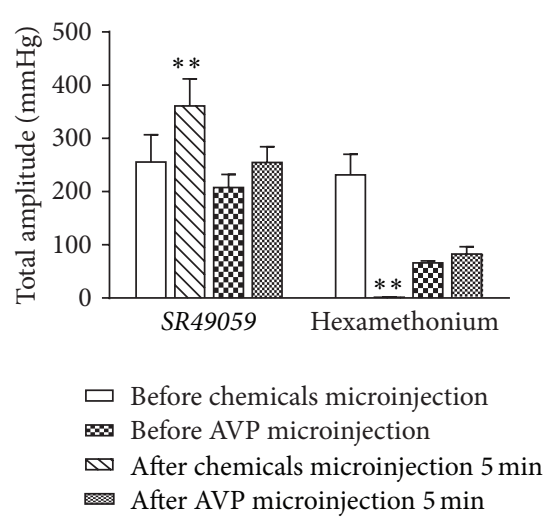

(b)

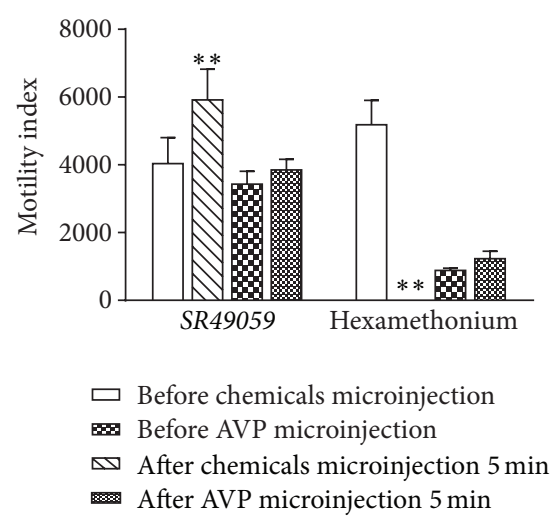

(c)

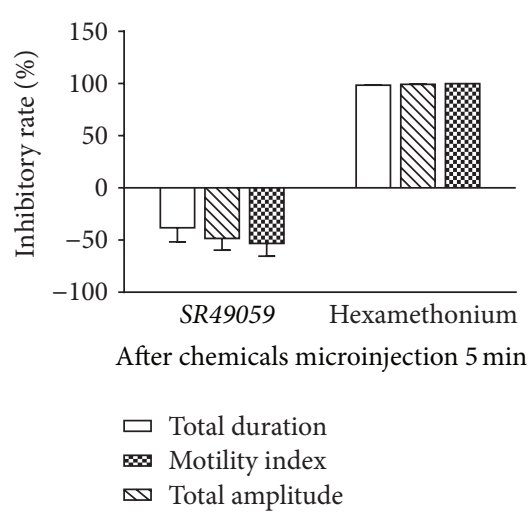

(d)

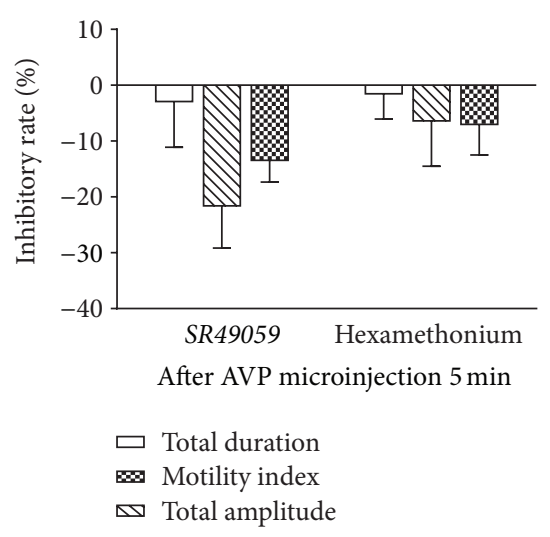

(e)

FIGURE 5: Effects of AVP (180 pmol) on gastric motility with pretreatment of SR49059 (320 pmol) or Hexamethonium $(8 \mu \mathrm{mol})$. Mean data of total duration (a) and total amplitude (b) and motility index (c) before microinjection and after microinjection 0-5 min, 5-10 min, and $10-15 \mathrm{~min}$. Average inhibitory rate of total duration, total amplitude, and motility index before and after microinjection of SR49059 (d) and Hexamethonium (e). ${ }^{*} P<0.05$ and ${ }^{* *} P<0.01$, versus before microinjection.

study, the microinjection sites were distributed in the rostral, middle, and caudal DMV regions. The microinjection of AVP into the whole DMV led to the inhibition of gastric motility. According to the previous results [11], there are AVP receptors in the soma and/or dendrite membranes of neurons in the whole DMV. Therefore, these findings suggest that the AVPsensitive neurons are wide spread all over the DMV.

4.2. Possible Mechanism of the Effect of AVP on Gastric Motility. The effect of AVP injected into the DMV on gastric motility was blocked by SR49059. This result was similar to previous findings in vitro [26], which reported that AVP $\left(10^{-6} \mathrm{M}\right)$ could increase the firing rate of a portion of neurons in the DMV and that the excitatory responses could be blocked completely by the selective V1 receptor antagonist. This result strongly suggests that AVP may inhibit the gastric motility by activating the specific AVP receptor in the DMV.

It has been known that the vagal preganglionic fibers originated from the DMV regulate a large component of gastric motility patterns via the enteric nervous system (ENS) and/or the network of pacemaker interstitial Cajal cells (ICC) within the muscle wall of the stomach. In the present study, the gastric contractions were completely inhibited by Hexamethonium (autonomic ganglionic cholinergic receptor blockade), which means that the AVP-sensitive neurons controlling gastric motility should be cholinergic. Thirty minutes later, the gastric contraction waves were restored but at a lower amplitude and no effect of AVP microinjected into the MDV on gastric motility was recorded by pretreatment with Hexamethonium. We hypothesize that a part of the vagal preganglionic fiber endings might establish synaptic contact with ICC and in turn control the activity of gastric smooth muscles under normal physiological state [27]. Hexamethonium blocked the signal transmission within the synapse, and the excitability of ICC was decreased temporarily by the sudden deficiency in controlling of vagal preganglionic fibers but gradually renewed after about 30 minutes. Thus, the restored waves with lower amplitude were largely mediated by nonneural mechanism, namely, were due to slow wave depolarization generated by pacemaker ICC.

Previous studies have demonstrated that the inhibitory pathways of vagal control of gastric function are composed of two types of preganglionic vagal neurons in the DMV. The first type consists of preganglionic cholinergic neurons, 
synapsed with postganglionic NANC neurons [23, 25, 28, 29], for example, nitrergic/VIPergic neurons in the gastric wall [30]. The second type is preganglionic nitrergic neurons, synapsed with postganglionic NANC neurons [16, 31]. Considering these data, it would be reasonable to assume that AVP plays a role in regulating gastric motility through the preganglionic cholinergic and postganglionic NANC neural pathway, rather than the preganglionic nitrergic and postganglionic NANC neural pathway, but more studies are needed to elucidate the specific types of postganglionic fibers.

\subsection{Effects of AVP on Respiratory Frequency, Heart Rate, and} Blood Pressure. It is also well known that the DMV contains the vagal parasympathetic preganglionic neurons innervating the heart $[32,33]$ and that the microinjection of OT into the DMV produces a consistent reduction in heart rate [20]. Similarly, in our study, the microinjection of $180 \mathrm{pmol}$ AVP into the DMV significantly inhibited the heart rate $(P<0.05)$. It has been also reported that transfusions of AVP could lead to an increase in airway resistance and a decrease in lung compliance and that AVP microinjected into the areas of the ventrolateral medulla (VLM) could inhibit the phrenic nerve activity and respiration in rat [34]. These results indicate that AVP might cause an inhibitory effect on respiration via both peripheral and central mechanisms. In our study, the effect of AVP injected into DMV on the respiratory frequency was consistent with these previous findings.

AVP is known to have hypertensive effects following intravenous infusion, and the changes of arterial blood pressure frequently alter the intragastric pressure accompanied by the gastric wall blood flow [35]. In this study, blood pressure remained almost constant during the microinjection of different doses of AVP into DMV. All these results suggest that the dosage of AVP is enough to activate the vagal preganglionic neurons in the DMV, and AVP-induced changes in gastric motility are likely attributed to central mechanism, rather than its hypertensive effects.

\section{Conflict of Interests}

The authors declare that there is no conflict of interests regarding the publication of this paper.

\section{Acknowledgments}

The authors would like to thank Professor Zong-ming Ren and Dr. Xiu-song Wang for technical assistance, and they also would like to thank Mogo Internet Technology Co., Ltd., for the professional English-language editing service. This work was financially supported by the National Science Foundation of China (no. 31071920) and the National Science Foundation of Shandong province (no. ZR2011CM029).

\section{References}

[1] L. Li, X. Kong, H. Liu, and C. Liu, "Systemic oxytocin and vasopressin excite gastrointestinal motility through oxytocin receptor in rabbits," Neurogastroenterology and Motility, vol. 19, no. 10, pp. 839-844, 2007.
[2] V. A. Tobin, H. Hashimoto, D. W. Wacker et al., "An intrinsic vasopressin system in the olfactory bulb is involved in social recognition," Nature, vol. 464, no. 7287, pp. 413-417, 2010.

[3] K. C. Bicego-Nahas, A. A. Steiner, E. C. Carnio, J. AntunesRodrigues, and L. G. S. Branco, "Antipyretic effect of arginine vasotocin in toads," American Journal of Physiology-Regulatory Integrative and Comparative Physiology, vol. 278, no. 6, pp. R1408-R1414, 2000.

[4] J. Schilling and F. Nürnberger, "Dynamic changes in the immunoreactivity of neuropeptide systems of the suprachiasmatic nuclei in golden hamsters during the sleep-wake cycle," Cell and Tissue Research, vol. 294, no. 2, pp. 233-241, 1998.

[5] G. Decaux, A. Soupart, and G. Vassart, "Non-peptide argininevasopressin antagonists: the vaptans," The Lancet, vol. 371, no. 9624, pp. 1624-1632, 2008.

[6] T. Hayakawa, S. Kuwahara-Otani, S. Maeda, K. Tanaka, and M. Seki, "Collateral projections of the dorsal motor nucleus of the vagus nerve to the stomach and the intestines in the rat," Okajimas Folia Anatomica Japonica, vol. 90, no. 1, pp. 7-15, 2013.

[7] C. J. Willett, J. G. Rutherford, D. G. Gwyn, and R. A. Leslie, "Projections between the hypothalamus and the dorsal vagal complex in the cat: an HRP and autoradiographic study," Brain Research Bulletin, vol. 18, no. 1, pp. 63-71, 1987.

[8] P. E. Sawchenko and L. W. Swanson, "Immunohistochemical identification of neurons in the paraventricular nucleus of the hypothalamus that project to the medulla or to the spinal cord in the rat," Journal of Comparative Neurology, vol. 205, no. 3, pp. 260-272, 1982.

[9] R. Landgraf, T. Malkinson, T. Horn, W. L. Veale, K. Lederis, and Q. J. Pittman, "Release of vasopressin and oxytocin by paraventricular stimulation in rats," American Journal of Physiology: Regulatory Integrative and Comparative Physiology, vol. 258, no. 1, part 2, pp. R155-R159, 1990.

[10] D.-Q. Zhao, C.-L. Lu, and H.-B. Ai, “The role of catecholaminergic neurons in the hypothalamus and medullary visceral zone in response to restraint water-immersion stress in rats," Journal of Physiological Sciences, vol. 61, no. 1, pp. 37-45, 2011.

[11] D.-Q. Zhao and H.-B. Ai, "Oxytocin and vasopressin involved in restraint water-immersion stress mediated by oxytocin receptor and vasopressin $1 \mathrm{~b}$ receptor in rat brain," PLoS ONE, vol. 6, no. 8, Article ID e23362, 2011.

[12] I. J. Llewellyn-Smith, D. O. Kellett, D. Jordan, K. N. Browning, and R. A. Travagli, "Oxytocin-immunoreactive innervation of identified neurons in the rat dorsal vagal complex," Neurogastroenterology and Motility, vol. 24, no. 3, pp. el36-e146, 2012.

[13] R. Babygirija, J. Zheng, K. Ludwig, and T. Takahashi, "Central oxytocin is involved in restoring impaired gastric motility following chronic repeated stress in mice," American Journal of Physiology: Regulatory Integrative and Comparative Physiology, vol. 298, no. 1, pp. R157-R165, 2010.

[14] D. Ocampo Daza, M. Lewicka, and D. Larhammar, “The oxytocin/vasopressin receptor family has at least five members in the gnathostome lineage, inclucing two distinct V2 subtypes," General and Comparative Endocrinology, vol. 175, no. 1, pp. 135-143, 2012.

[15] M. Raggenbass, "Overview of cellular electrophysiological actions of vasopressin," European Journal of Pharmacology, vol. 583, no. 2-3, pp. 243-254, 2008.

[16] S.-Y. Zhou, Y.-X. Lu, H. Yao, and C. Owyang, "Spatial organization of neurons in the dorsal motor nucleus of the vagus synapsing with intragastric cholinergic and nitric oxide/VIP neurons 
in the rat," American Journal of Physiology: Gastrointestinal and Liver Physiology, vol. 294, no. 5, pp. G1201-G1209, 2008.

[17] G. M. Holmes, K. N. Browning, T. Babic, S. R. Fortna, F. H. Coleman, and R. A. Travagli, "Vagal afferent fibres determine the oxytocin-induced modulation of gastric tone," The Journal of Physiology, vol. 591, no. 12, pp. 3081-3100, 2013.

[18] L. M. Flanagan, B. R. Olson, A. F. Sved, J. G. Verbalis, and E. M. Stricker, "Gastric motility in conscious rats given oxytocin and an oxytocin antagonist centrally," Brain Research, vol. 578, no. 1-2, pp. 256-260, 1992.

[19] M. J. McCann and R. C. Rogers, "Oxytocin excites gastricrelated neurones in rat dorsal vagal complex," Journal of Physiology, vol. 428, pp. 95-108, 1990.

[20] R. C. Rogers and G. E. Hermann, "Dorsal medullary oxytocin, vasopressin, oxytocin antagonist, and TRH effects on gastric acid secretion and heart rate," Peptides, vol. 6, no. 6, pp. 11431148, 1985.

[21] R. C. Rogers and G. E. Hermann, "Hypothalamic paraventricular nucleus stimulation-induced gastric acid secretion and bradycardia suppressed by oxytocin antagonist," Peptides, vol. 7, no. 4, pp. 695-700, 1986.

[22] D. M. Armstrong, L. Manley, J. W. Haycock, and L. B. Hersh, "Co-localization of choline acetyltransferase and tyrosine hydroxylase within neurons of the dorsal motor nucleus of the vagus," Journal of Chemical Neuroanatomy, vol. 3, no. 2, pp. 133-140, 1990.

[23] R. A. Travagli, G. E. Hermann, K. N. Browning, and R. C. Rogers, "Brainstem circuits regulating gastric function," Annual Review of Physiology, vol. 68, pp. 279-305, 2006.

[24] K. N. Browning and R. A. Travagli, "Plasticity of vagal brainstem circuits in the control of gastric function," Neurogastroenterology and Motility, vol. 22, no. 11, pp. 1154-1163, 2010.

[25] M. T. Cruz, E. C. Murphy, N. Sahibzada, J. G. Verbalis, and R. A. Gillis, "A reevaluation of the effects of stimulation of the dorsal motor nucleus of the vagus on gastric motility in the rat," American Journal of Physiology-Regulatory Integrative and Comparative Physiology, vol. 292, no. 1, pp. R291-R307, 2007.

[26] Z.-L. Mo, T. Katafuchi, H. Muratani, and T. Hori, "Effects of vasopressin and angiotensin II on neurones in the rat dorsal motor nucleus of the vagus, in vitro," The Journal of Physiology, vol. 458, pp. 561-577, 1992.

[27] S. M. Ward, E. A. H. Beckett, X. Wang, F. Baker, M. Khoyi, and K. M. Sanders, "Interstitial cells of Cajal mediate cholinergic neurotransmission from enteric motor neurons," Journal of Neuroscience, vol. 20, no. 4, pp. 1393-1403, 2000.

[28] R. A. Travagli, G. E. Hermann, K. N. Browning, and R. C. Rogers, "Musings on the wanderer: What's new in our understanding of vago-vagal reflexes? III. Activity-dependent plasticity in vago-vagal reflexes controlling the stomach," American Journal of Physiology-Gastrointestinal and Liver Physiology, vol. 284, no. 2, pp. G180-G187, 2003.

[29] H. Y. Chang, H. Mashimo, and R. K. Goyal, "Musings on the wanderer: What's new in our understanding of vago-vagal reflex? IV. Current concepts of vagal efferent projections to the gut," The American Journal of Physiology-Gastrointestinal and Liver Physiology, vol. 284, no. 3, pp. G357-G366, 2003.

[30] T. Takahashi and C. Owyang, "Vagal control of nitric oxide and vasoactive intestinal polypeptide release in the regulation of gastric relaxation in rat," Journal of Physiology, vol. 484, no. 2, pp. 481-492, 1995.

[31] Z. K. Krowicki, D. V. Sivarao, T. P. Abrahams, and P. J. Hornby, "Excitation of dorsal motor vagal neurons evokes non-nicotinic receptor-mediated gastric relaxation," Journal of the Autonomic Nervous System, vol. 77, no. 2-3, pp. 83-89, 1999.

[32] J. H. Hsieh, Y. C. Chang, J. L. Chung et al., "The relationship between FTL and NA, DMV or CVLM in central cardiovascular control," Chinese Journal of Physiology, vol. 44, no. 4, pp. 169179, 2001.

[33] X.-Y. Zhang, H.-B. Ai, and X.-Y. Cui, "Effects of nuclei ambiguus and dorsal motor nuclei of vagus on gastric $\mathrm{H}^{+}$and $\mathrm{HCO}_{3}^{-}$ secretion in rats," World Journal of Gastroenterology, vol. 12, no. 20, pp. 3271-3274, 2006.

[34] C.-W. Chuang, M.-T. Cheng, J.-T. Lin, H.-Y. Hsien, H.-Y. Hung, and J.-C. Hwang, "Arginine vasopressin produces inhibition upon respiration without pressor effect in the rat," Chinese Journal of Physiology, vol. 46, no. 2, pp. 71-81, 2003.

[35] S. E. Spencer and W. T. Talman, "Modulation of gastric and arterial pressure by nucleus tractus solitarius in rat," American Journal of Physiology-Regulatory Integrative and Comparative Physiology, vol. 250, no. 6, part 2, pp. R996-R1002, 1986. 


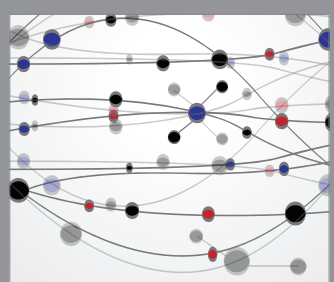

The Scientific World Journal
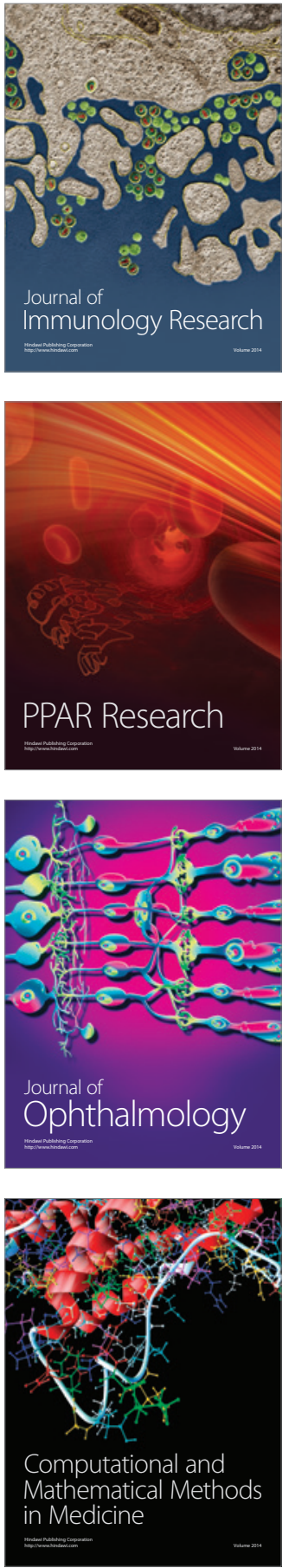

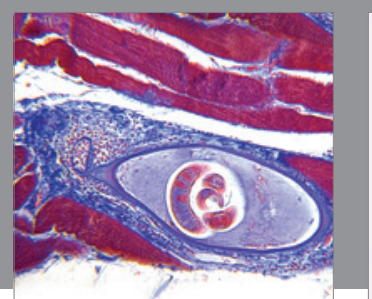

Gastroenterology Research and Practice

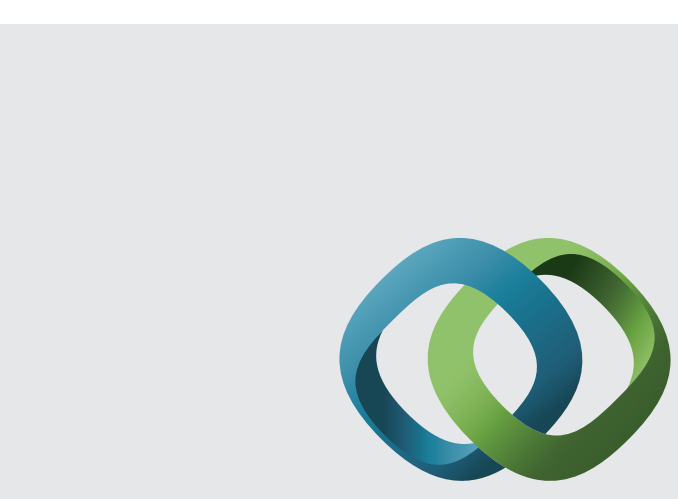

\section{Hindawi}

Submit your manuscripts at

http://www.hindawi.com
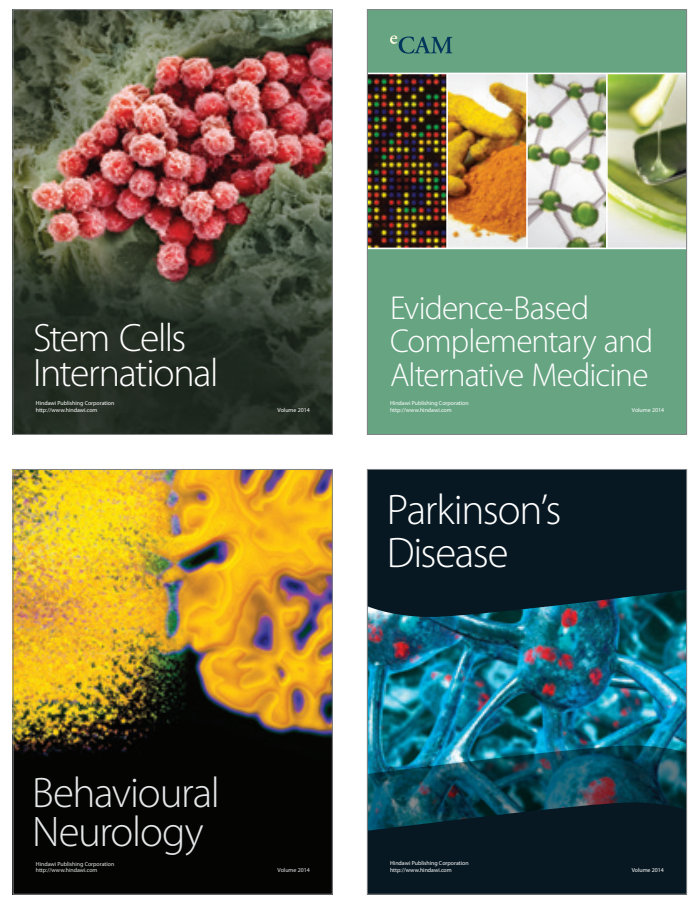
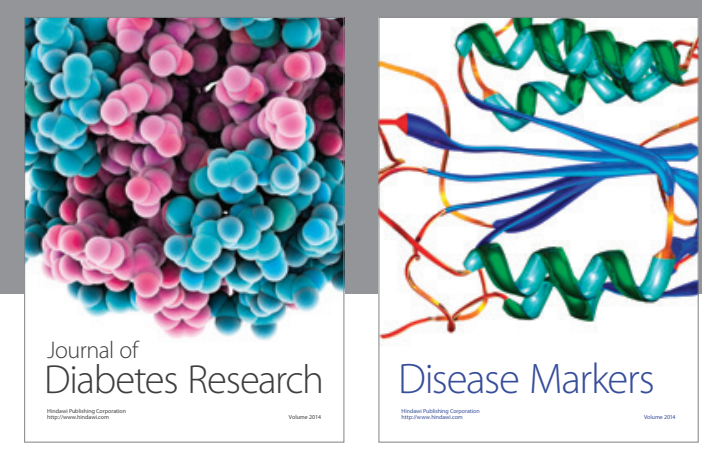

Disease Markers
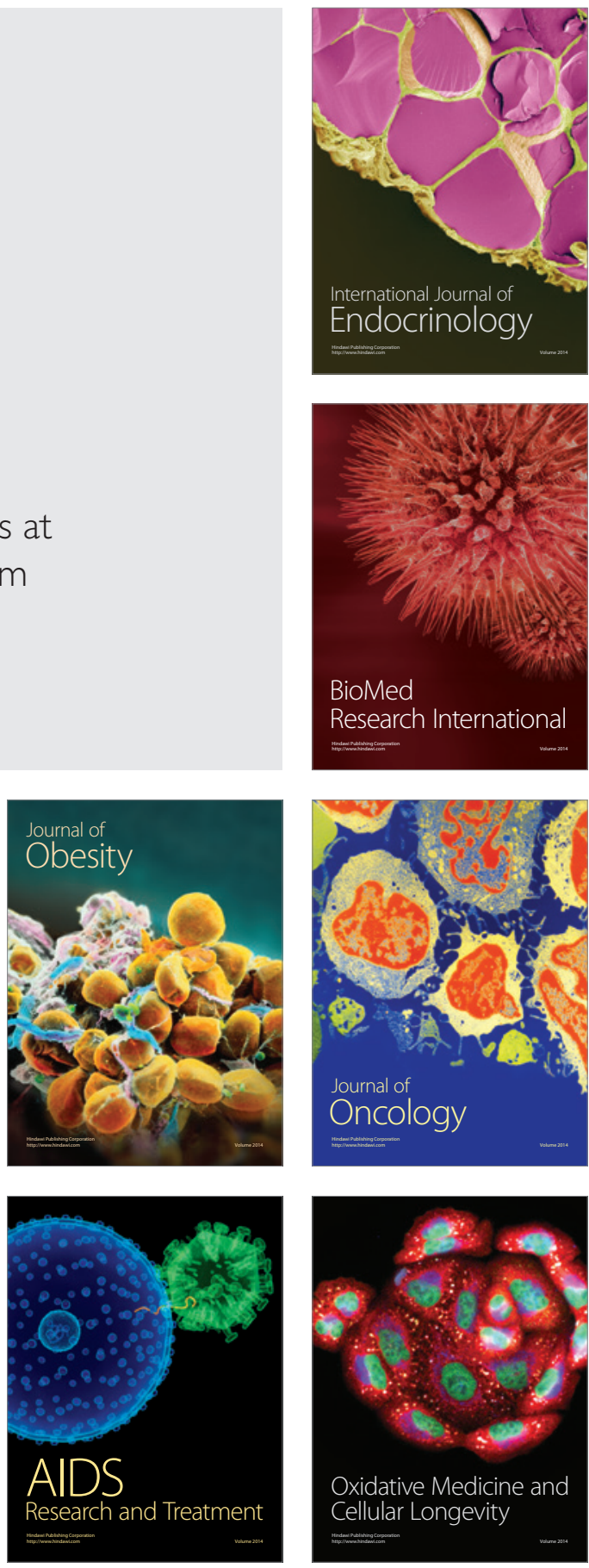